\title{
Peeling and Sliding in Nucleosome Repositioning
}

\author{
Tom Chou \\ Dept. of Biomathematics \& Dept. of Mathematics, UCLA, Los Angeles, CA 90095
}

(Dated: August 4, 2021)

\begin{abstract}
We investigate the mechanisms of histone sliding and detachment with a stochastic model that couples thermally-induced, passive histone sliding with active motor-driven histone unwrapping. Analysis of a passive loop or twist defect-mediated histone sliding mechanism shows that diffusional sliding is enhanced as larger portions of the DNA is peeled off the histone. The mean times to histone detachment and the mean distance traveled by the motor complex prior to histone detachment are computed as functions of the intrinsic speed of the motor. Fast motors preferentially induce detachment over sliding. However, for a fixed motor speed, increasing the histone-DNA affinity (and thereby decreasing the passive sliding rate) increases the mean distance traveled by the motor.
\end{abstract}

PACS numbers: 87.16.Sr,87.10.+e,87.16.Nn,87.14.Gg

The nucleosome is comprised of double-stranded DNA that wraps $1 \frac{3}{4}$ turns around the edge of disk-shaped histone proteins. These structures impart a DNA accessibility-based code in addition to the sequence-based genetic code [1]. Positioning of the histone particles depends on the local histone-DNA affinity (via $e$. g., DNA bendability [1]) and can modulate the accessibility of DNA. The remodeling of nucleosomes, involving perhaps histone sliding and/or detachment occurs during cellular processes that require DNA accessibility [2]. For example, in replication, translation of a replication fork along the DNA requires histones be either shifted or removed $[3,4]$. It has been proposed that these processes may be passive, in which parts of the histone-DNA complex thermally unwind and rewind, leaving transient regions of the DNA accessible $[2,3,5-7]$. Histone sliding has also been observed and modeled theoretically [8]. However, since the histone-dsDNA binding energy is $\sim 40 k_{B} T$, sliding mechanisms must exploit low energy thermal excitation such as the propagation of extra-length loops or twist defects [9]. Although these thermal mechanisms result in slow histone sliding, more rapid nucleosome remodeling can be catalyzed by molecular motor enzymes [10, 11]. A number of ATP-dependent remodeling factors, such as SWI/SNF, ISWI, and SWI2/SNF2 have been identified to be important during transcription $[4,6,12]$. The action of these remodeling factors may be required for more rapid detachment of histones from the DNA substrate. Apart from the observation that chromatin remodeling factors have conserved helicase-like ATPase domains [12], and that histones can both slide along and dissociate from DNA, there has been no explicit mechanistic hypothesis for enzymatically mediated chromatin remodeling.

In this Letter, we propose and explore the consequences of a simple, explicit mechanism for enzymemediated chromatin remodeling. The remodeling factor is modeled as a processing motor that runs into an isolated dsDNA-wrapped histone particle. Such a motor not only can bias histone sliding, inducing a drift, it can

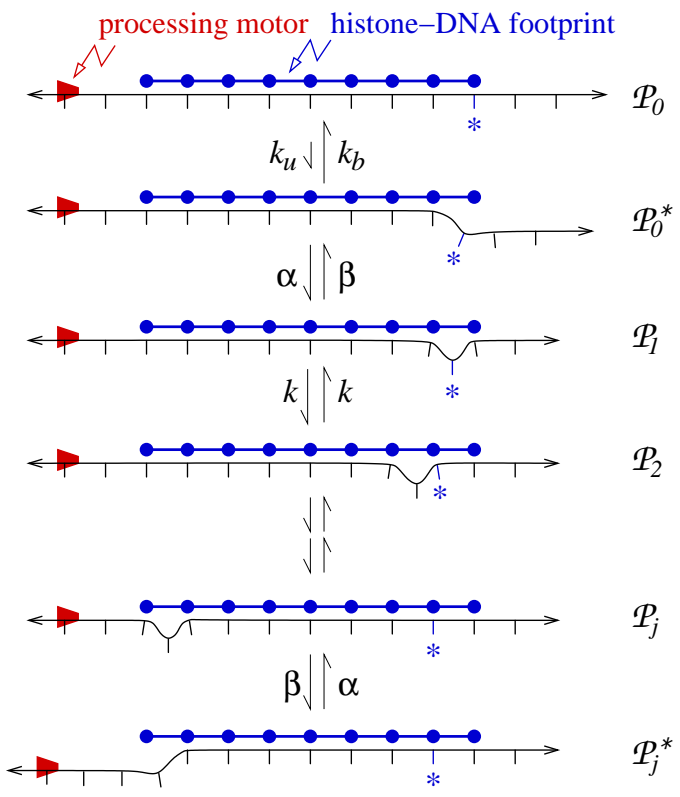

FIG. 1: The kinetics of single, isolated histone motion mediated by thermal creation and propagation of loops. Flaps unbind and bind at either end of the contact region with rate $k_{u}$ and $k_{b}$, respectively. Closure of a flap into a loop occurs at rate $\alpha$ and loop ejection into a flap occurs with rate $\beta$. Unbiased loop hopping rates in the interior of the contact region occurs with rate $k$. The asterisk labels a fixed site on the DNA substrate. A motor complex is shown to the left.

also wedge itself underneath the histone, peeling off the DNA. The full stochastic process is described by an effective master equation, from which we find the mean times to histone detachment, and the mean travel distance of the motor before it peels the histone off the DNA. Figure 1 depicts the histone-DNA contact region, or footprint, and a thermal inchworming mechanism. Small flaps (or defects) are thermally excited with rate $k_{u}$ by fluctuating segments of DNA that momentarily unwrap from the either edge of the histone-DNA contact region. The flap can rebind to the segment from which it detached with 
rate $k_{b}$, or, it can reattach to the next-nearest neighbor segment with rate $\alpha$, generating a loop. Once an interior loop is formed, it can hop to the right or left with rate $k$. The histone has translated one loop arclength only when a loop entering on one side of the contact region has left the opposite end. In this reptation-like mechanism, the net motion of an isolated histone is related to the statistics of diffusing loop "particles" that exit the end opposite from which they were thermally excited, analogous to the gambler's ruin problem [13]. The sites of the coarse-grained lattice represent individual histoneDNA contact "bond" segments, separated by a distance equivalent to the typical arclength of a flap, about 10 base pairs [3].

For a histone particle wrapped with $n$ segments containing a single interior loop, we denote the probability that the loop is at position $i(0 \leq i \leq n)$ by $\mathcal{P}_{i}$. Since the injection of loops is slow $\left(\alpha \ll k_{b}\right)[9,14]$, the probability for multiple loops is low, and loop-loop interactions can be neglected. The master equation governing the interior diffusive motion of the loop is $\dot{\mathcal{P}}_{i}=k \mathcal{P}_{i-1}+k \mathcal{P}_{i+1}-2 k \mathcal{P}_{i}$ for $2 \leq i \leq n-1$. Near the left edge the boundary conditions are $\dot{\mathcal{P}}_{1}=k \mathcal{P}_{2}-(\beta+k) \mathcal{P}_{1}+\alpha \mathcal{P}_{0}^{*}$ and $\dot{\mathcal{P}}_{0}^{*}=k_{u} \mathcal{P}_{0}-\left(k_{b}+\alpha\right) \mathcal{P}_{0}^{*}+\beta \mathcal{P}_{1}$. Similar expressions hold at the right edge. The probability that a loop exits the opposite end from which it entered can be found by considering a unit source of probability $\mathcal{P}_{0}^{*}=1$, and solving the resulting recursion equations [13]. The total probability of starting in state $\mathcal{P}_{0}^{*}$ and arriving in state $\mathcal{P}_{n}$ is $\frac{\alpha k}{(n-1) \beta^{2} k_{b}+\beta k\left(\alpha+2 k_{b}\right)}$. Since flaps are thermally generated with rate $k_{u}$, and produce the state $\mathcal{P}_{n}^{*}$ with rate $\beta \mathcal{P}_{n}$, the effective hopping rate of the entire histone along the DNA is

$$
p_{n}=\frac{\alpha k k_{u}}{(n-1) \beta k_{b}+k\left(\alpha+2 k_{b}\right)} .
$$

For an isolated, fully-wrapped histone, flap generation at each end is statistically independent and its motion is that of unbiased diffusion with diffusivity $p_{N}$. The loop hopping rate $k$ will be approximated as two sequential flap detachment and reattachment steps, without any additional barriers, giving $k \sim k_{u} k_{b} /\left(k_{u}+k_{b}\right)$. Similarly, $\beta$ can be approximated by the detachment rate. Finally, the insertion of a loop is proportional to the flap reattachment rate, but reduced by a factor associated with the bending required to form a loop. Thus, we use the physical rate estimates

$$
\alpha \approx \alpha_{0} k_{b}, \quad \beta \approx k_{u}, \text { and } k \approx k_{u} k_{b} /\left(k_{u}+k_{b}\right),
$$

where $\alpha_{0} \sim e^{-E_{b} / k_{B} T} \ll 1$ scales the loop injection rate by the energy $E_{b}$ required to bend the DNA into a small loop. These approximations make our results depend on just $w / \alpha$ and $K=k_{b} / k_{u}$, and the maximum number $N$ of DNA-histone contacts. Since $E_{b} \sim 23 k_{B} T[9,14]$ and $\alpha_{0} \sim 10^{-10}$, we present all results in terms of quantities rescaled to the slowest rate $\alpha$ in the problem.

Figure 2a shows the normalized effective hopping rate $p_{n} / \alpha$ as a function of $K=k_{b} / k_{u}$ and $n$. For fixed $k_{b}$,
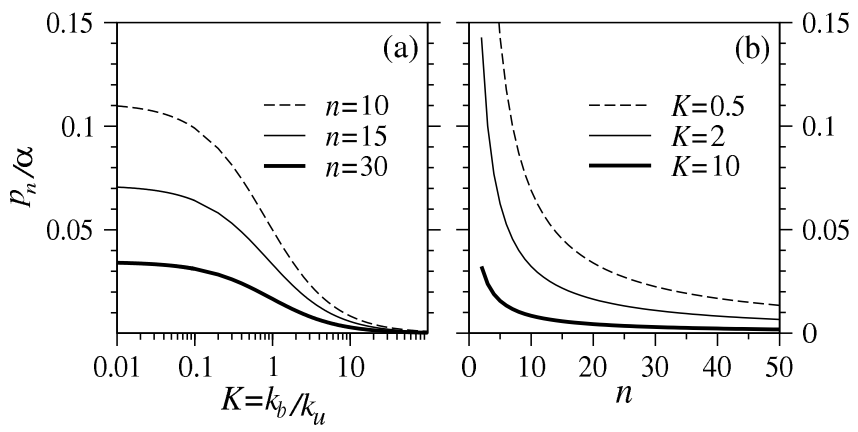

FIG. 2: (a) Effective diffusion coefficient $p_{n} / \alpha$ as a function of flap binding constant $K=k_{b} / k_{u}$. (b) Diffusivity as a function of the degree of histone-DNA wrapping $n$. The parameters used are $\alpha=10^{-10} k_{b}, \beta=k_{u}$, and $k=k_{b} k_{u} /\left(k_{b}+k_{u}\right)$ (Eq. $2)$.

$p_{n}$ decreases with decreasing flap unbinding rate $k_{u}$. The effective hopping rate also depends on the number $n$ of histone-DNA contact elements, as shown in Fig. 2b. The higher the degree of wrapping of a histone, the larger the $n$, and the smaller its loop-mediated diffusion coefficient.

Now consider a processing motor complex that moves unidirectionally with rate $w$ along the DNA. For simplicity, we assume that the motor moves by steps of length approximately equal to that of a thermally generated loop. Fig. 1 shows a motor to the left of a histoneDNA contact region. The motor can advance only if the segment in front of it is cleared and not attached to the histone. The edge of the contact region just ahead of a motor can be cleared by a thermally-excited flap, allowing the motor to possibly move forward and slip under it. Consequently, the effective forward hopping rate $\bar{w}$ of a motor that is peeling a histone is reduced from the free hopping rate $w$ by a factor dependent on the reattachment rate $k_{b}$ :

$$
\bar{w}=\frac{w k_{u}}{w+k_{b}} .
$$

The motor can also advance into the contact region by loops generated at the opposite end, but that have arrived after diffusing across the footprint. Thermal excitation of a flap at the far end, and subsequent propagation of a loop to the opposite end, occurring with rate $p_{n}$, momentarily creates a flap of two bond segments near the motor. Again, flap closing competes with motor insertion underneath the flap. As shown in Fig. 3, there are three fates available to a two-bond flap nearest the motor. The motor can remain in its position and both bonds 


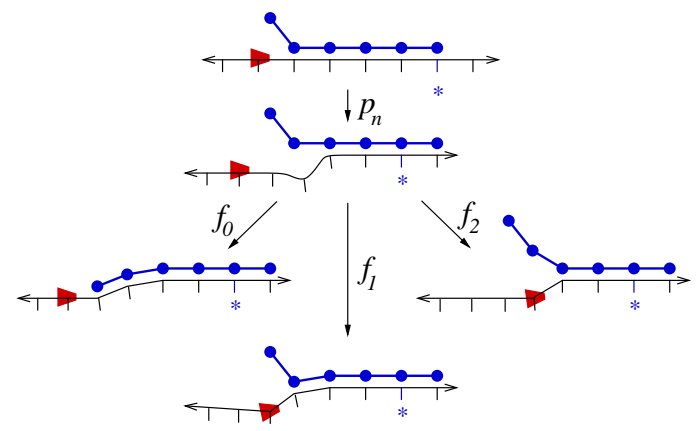

FIG. 3: Details of the motor-flap interaction. When a loop generated on the opposite end exits the side nearest the motor (with effective rate $p_{n}$ ), the motor can move forward 0,1 , or 2 steps with relative probabilities $f_{0}, f_{1}$, and $f_{2}$, respectively.

of the flap can close (with effective rate $k_{b} / 2$ ), the motor can move one position forward and one bond of the flap can close (with effective rate $w k_{b} /\left(w+k_{b}\right)$ ), or, the motor can move forward two sites (with effective rate $w / 2$ ) under the flap, preventing it from closing at all. The total transition rate available to the two-bond flap is $r_{T}=k_{b} / 2+k_{b} w /\left(k_{b}+w\right)+w / 2$, from which we find the probabilities for each pathway

$$
f_{0}=\frac{k_{b}}{2 r_{T}}, f_{1}=\frac{k_{b} w}{r_{T}\left(k_{b}+w\right)}, \text { and } f_{2}=\frac{w}{2 r_{T}} .
$$

Note that $f_{0}+f_{1}+f_{2}=1$ and that the ratios of these pathways are determined by the motor speed $w$. These probabilities apply only to flaps on the motor side of the contact region. On the opposite, motor-free end, we assume that single bond flaps close sufficiently fast $(K \equiv$ $k_{b} / k_{u} \gg 1$ ) such that spontaneous desorption of histones is negligible in the absence of a processing motor.

As the progressing motor partially unwraps the histone, the length $n$ of the contact region decreases, increasing the effective sliding rate $p_{n}$ of the remaining contact region (cf. Fig. 2b). The sliding mechanism becomes increasingly important as the degree of unpeeling increases, and the histone becomes more efficient at diffusively escaping the motor. If $m$ is the position of the motor, and $n$ is its distance to the far end of the footprint, a master equation that incorporates all of the kinetics described above can be written for the probability $P(m, n, t)$ :

$$
\begin{aligned}
& \dot{P}(m, n, t)= p_{N}[P(m, n+1)-2 P(m, n)+P(m, n-1)]+w[P(m-1, n+1)-P(m, n)], \quad n \geq N+1 \\
& \dot{P}(m, N, t)=-\left(\bar{w}+p_{N}\right) P(m, N)+w P(m-1, N+1)+f_{0} p_{N-1} P(m, N-1)+f_{1} p_{N} P(m-1, N)+p_{N} P(m, N+1), \\
& \dot{P}(m, n, t)=-\left(\bar{w}+p_{n}\right) P(m, n)+\bar{w} P(m-1, n+1)+f_{0} p_{n-1} P(m, n-1)+f_{1} p_{n} P(m-1, n) \\
&+f_{2} p_{n+1} P(m-2, n+1), \quad 3 \leq n<N,
\end{aligned}
$$

with $\dot{P}(m, 1)=-k_{u} P(m, 1)+\bar{w} P(m-1,2)+f_{2} p_{2} P(m-$ $2,2)$ and $\dot{P}(m, 2)=-\left(\bar{w}+p_{2}\right) P(m, 2)+\bar{w} P(m-1,3)+$ $f_{1} p_{2} P(m-1,2)+f_{2} p_{3} P(m-2,3)$. The statistics of the histone detachment time can be found by considering only the relative distance $n$, and when it first reaches $n=0$. Upon summing the time-integrated probabilities over all possible motor positions $m \geq 0$, Eqs. 5 are succinctly expressed as

$$
a_{n+1} Q_{n+1}-\left(a_{n}+b_{n}\right) Q_{n}+b_{n-1} Q_{n-1}=-\delta_{n, N}
$$

where $Q_{n} \equiv \int_{0}^{\infty} \mathrm{d} t \sum_{m=0}^{\infty} P(m, n, t)$ and $\delta_{n, N}=$ $\sum_{m=0}^{\infty} P(m, n, 0)$ is the initial condition with the motor at the left end of the contact region of a fully wrapped histone. In Eq. 6, the transition rates are

$$
\begin{array}{ll}
a_{n}=w+p_{N}, \quad b_{n}=p_{N} \quad & n>N \\
a_{n}=\bar{w}+f_{2} p_{n}, \quad b_{n}=f_{0} p_{n} & 2 \leq n \leq N .
\end{array}
$$

Conditions just prior to detachment $(n=1)$ also require $b_{0}=b_{1}=0$ and $a_{1}=k_{u}$.

The mean first detachment time of the process described by Eq. 6 is found by solving the corresponding Backward Kolmogorov equation [13], or by directly timeintegrating the solution $\left\langle T_{d}\right\rangle=\sum_{n=1}^{\infty} \int_{0}^{\infty} Q_{n}(t) \mathrm{d} t$ :

$$
\left\langle T_{d}\right\rangle=\sum_{n=1}^{N} \frac{1}{a_{n}}+\sum_{n=1}^{N} \sum_{i=1}^{\infty} \frac{1}{a_{n+i}} \prod_{k=0}^{i-1} \frac{b_{n+k}}{a_{n+k}} .
$$

The mean distance $\left\langle m_{d}\right\rangle$ traveled by the motor before it detaches the histone can be constructed from the timeintegrated quantity $R_{n} \equiv \int_{0}^{\infty} \mathrm{d} t \sum_{m=0}^{\infty} m P(m, n, t)$, which obeys an equation similar to (6): $a_{n+1} R_{n+1}-$ $\left(a_{n}+b_{n}\right) R_{n}+b_{n-1} R_{n-1}=G_{n}$. The additional inhomogeneity terms $G_{n>N}=-Q_{n}, G_{N}=-w Q_{N+1}$, and $G_{n \leq N}=-\left(\bar{w}+2 f_{2} p_{n+1}\right) Q_{n+1}-f_{1} p_{n} Q_{n}$, rely on the solution $Q_{n}$ to Eq. 6. Upon solving for $R_{n}$, we find 


$$
\left\langle m_{d}-1\right\rangle=k_{u} \int_{0}^{\infty} \mathrm{d} t \sum_{m=0}^{\infty} m P(m, 1, t)=k_{u} R_{1} .
$$

In Fig. 4a we plot the mean first detachment times as a function of the motor speed, for $N=15$ and various flap binding constants $K=k_{b} / k_{u}$. For slow motors, the double flaps near the motor nearly always close $\left(f_{0} \rightarrow 1\right)$ before the motor can advance. The histone unit diffuses along the DNA with rate $p_{N}$ and is only occasionally blocked by a relatively stationary motor. It spends most of its time away from the motor, preventing it from slipping under flaps that momentarily open. The resulting mean first detachment time diverges exponentially in the $w / \alpha \rightarrow 0$ limit.

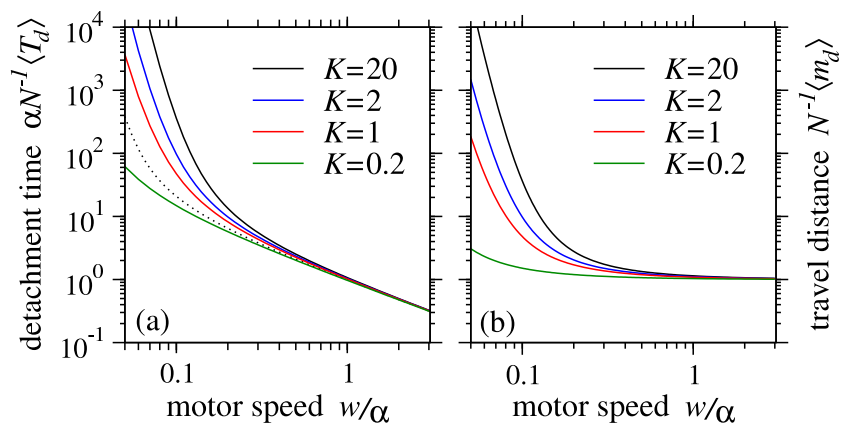

FIG. 4: (a) The normalized mean time to detachment, $\alpha\left\langle T_{d}\right\rangle / N$, as a function of the normalized motor speed $w / \alpha$, for various $K$. The dotted curve shows the dramatic decrease in detachment times corresponding to $K=20$ and a hypothetically fixed small diffusivity $p_{N}$ that suppresses sliding. (b) The mean distance $\left\langle m_{d}\right\rangle$ traveled by the motor before it peels off the histone. In both plots, the maximum wrapping length was set to $N=15$, corresponding to a flap/loop length of about 10 base pairs. Since we have assumed tight binding (large $K$ ), and ignored spontaneous histone desorption, our results $\left\langle T_{d}\right\rangle$ and $\left\langle m_{d}\right\rangle$ for smaller values of $K$ are only upper bounds.

In the large $w / \alpha$ limit, the remodeling complex slips under the flaps before loops can propagate through the contact region and the peeling mechanism dominates. As $w$ is increased, the peeling time decreases. In the extremely fast limit $w / k_{b} \gg 1$, the motor nearly always inserts itself underneath flaps before they can reattach $\left(f_{0} \approx f_{1} \approx 0\right.$, and $\left.f_{2} \approx 1\right)$ and Eq. 8 simplifies to $\left\langle T_{d}\right\rangle=k_{u}^{-1}+\sum_{n=2}^{N}\left(k_{u}+p_{n}\right)^{-1}<N k_{u}^{-1}$. The mean detachment time of a histone subjected to an infinitely fast motor would be $\left\langle T_{d}\right\rangle=N / k_{u}$ if flaps originated only in front of the motor. However, flap generation at, and loop propagation from the other end of the contact region enhances the arrival of flaps near the motor, thereby decreasing $\left\langle T_{d}\right\rangle$ to values below $N / k_{u}$.

In Fig. $4 \mathrm{~b}$, we plot the mean distance traveled by the motor before it detaches a histone, as a function of $w / \alpha$ for various $K$. For low motor speeds, sliding dominates peeling, and the motor moves significantly. Note as the flap binding affinity $K$ increases, the mean distance traveled by the motor also increases. For fixed $k_{b}$, increasing $K$ by decreasing $k_{u}$ leads to a dramatic increase in $\left\langle T_{d}\right\rangle$, especially at low motor speeds. This extra time allows the histone to slide a longer distance before being peeled off by the motor. Although increasing $K$ reduces the effective sliding rate of an isolated histone, mechanistically, the increase in mean distance traveled arises from the relative suppression of double flaps near the motor, slowing down the peeling process.

For larger $w / \alpha$, peeling becomes dominant and the motor moves a mean distance $\left\langle m_{d}\right\rangle \approx N$ before scrapping the entire histone off. Again, in the $w / k_{b} \gg 1$ limit, an analytic form can be explicitly found: $\left\langle m_{d}\right\rangle / N=$ $1+\sum_{n=2}^{N} p_{n} /\left(k_{u}+p_{n}\right)$. This slow increase in the normalized mean distance traveled, $\left\langle m_{d}\right\rangle / N$, with $N$ arises from the relative reduction of double flaps near the motor, thereby diminishing the frequency of this efficient mode of peeling. This reduction occurs for larger $N$ through the loop propagation probability $p_{n}$.

In summary, we have presented and analyzed a stochastic model for ATP-dependent chromatin remodeling that incorporates a competition between sliding and peeling of a single isolated histone. Our main findings include formulae for the mean detachment time and travel distance, as functions of motor speed and binding affinity. Histones with larger binding affinity to the DNA substrate slide farther before being peeled off by the motor. We have neglected effects such as DNA sequence dependence (which might affect $w, k_{u}, k_{b}$, and $k$ ) and twist defect mobility [3]. This latter mechanism may only open much smaller end flaps that allow motor insertion and peeling. Nonetheless, our results are useful for dissecting and quantifying in vitro measurements of passive sliding and ATPase-assisted nucleosome remodeling, particularly when physical chemical conditions can be controlled and $w, k_{u}, k_{b}$ tuned.

Nucleosomes in vivo typically consists of an array of interacting histones $[11,15]$. Thus, our results would be valid only if the typical DNA linker length $\ell$ between two adjacent histones were larger than $\left\langle m_{d}\right\rangle+N$. If $\ell \leq\left\langle m_{d}\right\rangle+N$, the histones would be squeezed together by the motor. In such cases, peeling would be enhanced as the sliding mobility is reduced due to histone-histone exclusion. Finally, it should be mentioned that the analysis of Eq. 5 is directly applicable to the study of dehybridization and driven translocation of double-stranded, mononucleotide nucleic acids through nanopores [16]. Our results, after reidentification of the parameters, provide an analytic solution to the mean passage times of translocation in the high driving force limit.

This work was supported by the NSF (DMS-0349195) and by the NIH (K25AI41935). 
[1] E. Segal et al. Nature, 442, 772, (2006).

[2] A. Saha, J. Wittmeyer, and B. R. Cairns, Nature Reviews, Mol. Cell Biol., 7, 437, (2006).

[3] H. Schiessel, J. Widom, R. F. Bruinsma, and W. M. Gelbart Phys. Rev. Lett., 86, 4414, (2001); 88, 129902, (2002).

[4] I. L. de la Serna and A. N. Imbalzano, Nature Genetics, 32, 560, (2002).

[5] G. Li, M. Levitus, C. Bustamante, and J. Widom, Nature Struct. \& Mol. Biol., 12, 46, (2005); J. Widom, Q. Rev. Biophys., 34, 269, (2001).

[6] G. Längst and P. B. Becker, J. Cell. Sci., 114, 2561, (2001); P. B. Becker, EMBO Journal, 21, 4749, (2002).

[7] W. Möbius, R. A. Neher, and U. Gerland, Phys. Rev.
Lett. 97, 208102, (2006).

[8] H. Schiessel, J. Phys. Condens. Matter, 15, R699, (2003).

[9] I. M. Kulić and H. Schiessel Phys. Rev. Lett., 91, 148103, (2003).

[10] I. Whitehouse, et al., Mol. \& C Cell. Biol., 23, 1935, (2003).

[11] J. K. Tyler, Eur. J. Biochem., 269, 2268, (2002).

[12] C. L. Smith, Functional and Structural Analysis of the Yeast SWI/SNF Complex: a Dissertation, Ph.D. Thesis, University of Massachusetts Medical School, 2004.

[13] S. Redner, A Guide to First-Passage Processes, (Cambridge University Press, 2001); P. A. Pury and M. O. Cáceres, J. Phys. A, 36, 2695, (2003).

[14] F. Mohammad-Rafiee, I. M. Kulić, and H. Schiessel, J. Mol. Biol., 344, 47-58, (2004).

[15] T. Chou, Europhys. Lett., 5, 753, (2003).

[16] G. Lakatos, et al., Phys. Biol., 2, 166, (2005). 